\title{
CORRECTION
}

View Article Online

View Journal | View Issue

\section{Correction: Real-space evidence of the equilibrium ordered bicontinuous double diamond structure of a diblock copolymer}

Cite this: Soft Matter, 2015, 11,4142

DOI: $10.1039 / \mathrm{c5sm} 90077 \mathrm{~h}$

www.rsc.org/softmatter

\author{
C. Y. Chu, ${ }^{a}$ X. Jiang, ${ }^{b}$ H. Jinnai, ${ }^{\star b}$ R. Y. Pei, ${ }^{a}$ W. F. Lin, ${ }^{c}$ J. C. Tsai ${ }^{c}$ and H. L. Chen ${ }^{\star a}$
}

Correction for 'Real-space evidence of the equilibrium ordered bicontinuous double diamond structure of a diblock copolymer' by C. Y. Chu et al., Soft Matter, 2015, 11, 1871-1876.

The authors would like to correct some errors in eqn (5), (6) and (7). Eqn (5) should be corrected as

$$
N_{\text {node, } \mathrm{D}}=\frac{N_{\mathrm{D}}}{2}
$$

Eqn (6) should be corrected as

$$
N_{\text {node, } \mathrm{G}}=\frac{2 N_{\mathrm{G}}}{3}
$$

Eqn (7) should be corrected as

$$
\frac{d_{110}}{d_{211}}=\sqrt{3} \times \sqrt[3]{\frac{2 N_{\text {node }, \mathrm{D}}}{N_{\text {node } \mathrm{G}}}}
$$

The theoretical value of the ratio of the domain spacing of OBDD structure to that of OBDG, $\frac{d_{110}}{d_{211}}$, calculated by the corrected eqn (7) becomes 1.09. The experimentally observed value of $\frac{d_{110}}{d_{211}}(=1.06)$ obtained from the ratio of the positions of the corresponding SAXS peaks is now close to that predicted theoretically by the corrected eqn (7), which indicates that the OBDD and the OBDG structure formed in the present SPP- $b$-PS system correspond to the thermodynamically equilibrium state at lower

and higher temperature, respectively.
It should be noted that the ratio of the lattice parameter of OBDG to that of OBDD $\left(\right.$ i.e, $\left.\frac{a_{\mathrm{G}}}{a_{\mathrm{D}}}\right)$ derived by eqn (4) in the paper via the corrected eqn (7) is 1.589; this value is close to that (i.e., 1.576) predicted based on the Bonnet transformation between OBDD and OBDG reported by Hyde et al. ${ }^{1}$ Our model is however more general in the sense that it is based on the assumption of volume conservation, while the Bonnet transformation assumes the conservation of Gaussian curvature, with the transformation between OBDD and OBDG preserving all angles, distances and areas on the surface.

The Royal Society of Chemistry apologises for these errors and any consequent inconvenience to authors and readers.

\section{References}

1 S. T. Hyde and S. Andersson, Differential geometry of crystal structure descriptions, relationships and phase transformation, Zeitschrift für Kristallographie, 1985, 170, 225.

\footnotetext{
${ }^{a}$ Department of Chemical Engineering, Frontier Research Center on Fundamental and Applied Sciences of Matters, National Tsing Hua University, Hsin-Chu 30013, Taiwan.E-mail: hlchen@che.nthu.edu.tw

${ }^{b}$ Institute for Materials Chemistry and Engineering (IMCE), Kyushu University, 744 Motooka, Nishi-ku, Fukuoka 819-0395, Japan. E-mail: hjinnai@cstf.kyushu-u.ac.jp

${ }^{c}$ Department of Chemical Engineering, National Chung Cheng University, Chia-Yi 62102, Taiwan
} 